\title{
The Sentimental Analysis for E-Commerce Application
}

\author{
V.Geetha, C.K.Gomathy, P.Manojkumar, N.S.L.S.V.Manohar
}

\begin{abstract}
Right now, characterize every one of those viewpoints as a component of item, and present a multi-dimensional feeling investigation approach for E-business audits. Specifically, we utilize an assessment dictionary growing system to evacuate the word uncertainty among various measurements, and propose a calculation for estimation investigation on $E$-business audits dependent on rules and a dimensional feeling vocabulary. Make word net lexicon: In this sort of archive, every single positive word are worked out independently and every negative word are worked out at one spot. Extraction of dataset: First dataset of openly accessible item audits were downloaded from the web and afterward the entry extraction structure recognizes significant areas of the content which is generally illustrative of the substance of the record. All the more explicitly, this progression includes distinguishing and extricating those particular item includes and the assessments on them.
\end{abstract}

Keywords - Early reviewer, Early review, Embedding model.

\section{INTRODUCTION}

Internet based life site is characterized as "a site that encourages meeting individuals, discovering like personalities, imparting and sharing substance, and building network"; this sort of site permits or empowers different kinds of exercises, for example, business, social, or a mix of the two. Web based life classes incorporate advanced library, web based business, diversion, audit and interpersonal organization. Informal community is the types of online networking,[1] Online life are social channels of correspondence utilizing electronic advancements, work stations, and versatile innovations. These advancements make profoundly intuitive stages through which people, networks, and associations can share data, examine, rate, remark, and alter client created and online substance. These progressions empower correspondence among organizations, associations, networks, and people. Online life advancements[2] change the manner in which people and enormous associations impart, and they are progressively being created. Wide scope of utilizations in business and open strategy utilizes assumption investigation. Nostalgic investigation is currently being utilized from explicit item showcasing to solitary conduct acknowledgment. companies have consistently[3] been worried about the general population. Notwithstanding papers, there are a wide scope of obstinate articles are listed in the web and other web-based social networking.

Revised Manuscript Received on June 30, 2020.

* Correspondence Author

Dr. V.Geetha*, Assistant Professor, CSE Department, SCSVMV Deemed to be University.

Dr. C.K.Gomathy, Assistant Professor, CSE Department, SCSVMV Deemed to be University

P.Manojkumar, UG Scholar CSE Department, SCSVMV Deemed to be University

N.S.L.S.V.Manohar, UG Scholar CSE Department, SCSVMV Deemed to be University.

(C) The Authors. Published by Blue Eyes Intelligence Engineering and Sciences Publication (BEIESP). This is an open access article under the CC BY-NC-ND license (http://creativecommons.org/licenses/by-nc-nd/4.0/)
Fine-grained supposition examination is an incredibly testing issue as a result of the assortment of manners by which conclusions can be communicated. News stories present a considerably more noteworthy test, as they normally dodge unmistakable markers of mentalities. Notwithstanding, regardless of their clear lack of bias, news stories[6] can in any case positive or negative. Numerous strategies utilized for opinion examination include various methods dependent on recognizing certain watchwords[7]. We use gullible performs fine-grained opinion investigation to order sentences as positive, negative or unbiased

\section{LITERATURE REVIEW}

These days Digital Libraries 2.0 are chiefly founded on the cooperation between clients through community oriented applications, for example, wikis, web journals, and so forth or new potential ideal models like the waves proposed by Google. This new idea, the wave, speaks to a typical space where assets and clients can cooperate. The issue emerges when the quantity of assets and clients is high, at that point apparatuses for helping the clients in their data needs are fundamental. Right now fluffy etymological recommender framework dependent on the Google Wave abilities is proposed as apparatus for conveying scientists inspired by normal research lines. The framework permits the making of a typical space by implies a wave as a method for teaming up and trading thoughts between a few analysts keen on a similar point. Furthermore,[8] the framework recommends, in a programmed way, a few scientists and helpful assets for each wave. These suggestions are processed after a few recently characterized inclinations and qualities by methods for fluffy semantic names. In this way the framework encourages the potential joint efforts between multidiscipliner[2] specialists and prescribes integral assets valuable for the association. A model of the framework has been created and tried with a few research bunches from a similar college accomplishing victories. A half and half fluffy based customized recommender framework for telecom items /administrations. The [3]Internet makes brilliant open doors for organizations to offer customized online types of assistance to their clients. Recommender frameworks are intended to naturally create customized recommendations of items/administrations to clients. Since different vulnerabilities exist inside both item and client information, it is a test to accomplish high suggestion precision. This examination builds up a half and half proposal approach which joins client based and thing based communitarian sifting systems with fluffy set strategies and applies it to versatile item and administration suggestion.

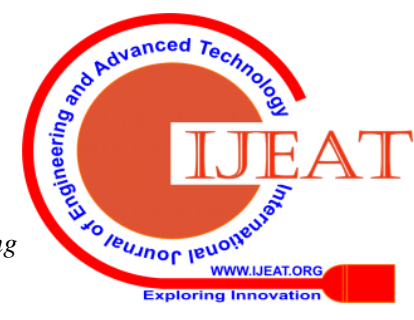




\section{The Sentimental Analysis for E-Commerce Application}

It especially actualizes the approach in framework programming. Trial results exhibit the viability of the proposed approach and the underlying application shows that the FTCP-RS can successfully assist clients with selecting the most appropriate portable items or administrations.

Recommender frameworks dependent on informal organizations[4] The conventional recommender frameworks, particularly the shared separating recommender frameworks, have been contemplated by numerous analysts in the previous decade. In any case, they disregard the social connections among clients. Truth be told, these connections can improve the exactness of proposal. As of late, the investigation[5] of social-based recommender frameworks has become a functioning examination point. Right now, propose a social regularization approach that joins interpersonal organization data to profit recommender frameworks. The two clients' companionships and rating records (labels) are utilized to anticipate the missing qualities (labels) in the client thing network. Particularlywe utilize a biclustering calculation to recognize the most reasonable gathering of companions for producing distinctive last proposals. Experimental examinations on genuine datasets show that the proposed approach accomplishes better execution than existing methodologies.

The requirements forever long learning and the fast improvement of data advances. In[7] online limited soundness and metacognition are two difficult problems, particularly when students face data over-burden and there is no information authority inside the learning condition. The half and half calculation can give more precise suggestions than big name.The proposed framework can encourage the arrangement customization in the networks learning.

RecomMetz: A setting mindful information based portable recommender framework for film showtimes

Recommender frameworks are utilized to give separated data from a lot of components. They give customized suggestions on items or administrations to clients. The suggestions are planned to give intriguing components[8] to clients. Recommender frameworks can be created utilizing various strategies and calculations where the choice of these methods relies upon the zone wherein they will be applied. This paper proposes a recommender framework in the recreation area, explicitly in the film showtimes space. The framework proposed is called RecomMetz, and it[9] is a setting mindful versatile recommender framework dependent on Semantic Web advances. In detail, an area cosmology basically serving a semantic closeness metric changed in accordance with the idea of "bundles of single things" was created right now. Moreover, area, group and time were considered as three various types of relevant data in RecomMetz. More or less, RecomMetz has special highlights: (1) the things to be suggested have a composite structure (cinema + film + showtime), (2) the mix of the time and group factors into a setting mindful model, (3) the usage of a cosmology based setting demonstrating approach and (4) the improvement of a multi-stage local versatile UI expected to use the equipment abilities (sensors) of cell phones. The assessment results show the productivity and adequacy of the suggestion component executed by
RecomMetz in both a cool beginning situation and a no chilly beginning situation. A tale cross breed approach improving viability of recommender frameworks. Recommender frameworks bolster clients by creating conceivably fascinating proposals[9] about pertinent items and data. The expanding consideration towards such apparatuses is seen by both the incredible number of amazing and complex recommender calculations created lately and their selection in numerous mainstream Web stages. Nonetheless, exhibitions of recommender frameworks can be influenced by numerous basic issues with respect to example, over-specialization, trait choice and adaptability. To moderate some of such negative impacts, a mixture recommender framework, called Relevance Based Recommender, is proposed right now. It misuses singular proportions of apparent importance processed by every client for each case of intrigue and, to acquire a superior exactness, likewise by considering the[21] closely resembling measures figured by different clients for similar occasions. A few tests show the points of interest presented by this recommender while producing conceivably appealing recommendations. A recommender framework for analysts dependent on bibliometrics .Recommender frameworks (RSs) abuse past practices and client likenesses to give customized proposals. There are a few points of reference of utilization in scholastic conditions to help clients finding pertinent data, in view of suspicions about the qualities of the things and clients. Regardless of whether quality has just been considered as a property of things in past works, it has never been given a key job in the repositioning procedure for the two things and clients. Right now, present before, a quality-based fluffy etymological Recommender framework for researchers. We propose the utilization of some bibliometric quantifies as the best approach to evaluate the nature of the two things and clients without the association of specialists just as the utilization of 2-tuple semantic way to deal with portray the phonetic data. The framework considers the deliberate quality as the primary factor for the re-positioning of the top-N suggestions list so as to call attention to analysts to the most recent and the best papers in their examination fields. To demonstrate the precision improvement, we lead an investigation including distinctive proposal draws near, targeting estimating their exhibition gain. The outcomes acquired end up being palatable for the analysts from various offices who partook on the tests.

A distributed recommender framework for self-developing client networks dependent on tattle overlays

Tattle based shared conventions end up being productive for supporting dynamic and complex data trade among disseminated peers. They are helpful for building and keeping up the system topology itself just as to help an inescapable dissemination of the data infused into the system. This is exceptionally valuable in our current reality where there is a developing need to get to and know about numerous sorts of disseminated assets like Internet pages, shared records, online items, news and data.

Published By:
Blue Eyes Intelligence Engineering

\& Sciences Publication

(C) Copyright: All rights reserved.

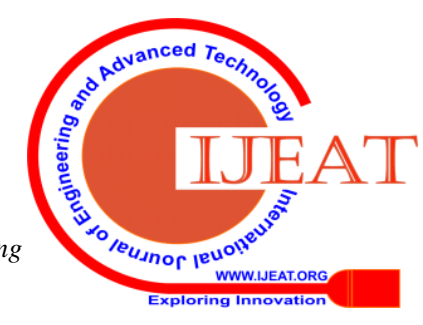


Finding adaptable, versatile and effective components tending to this theme is a key issue, even with applicable social and financial angles. Right now, propose the general design of a framework whose point is to misuse the synergistic trade of data between peers so as to construct a framework ready to accumulate comparable clients and spread helpful recommendations among them. Also, the launch of a recommender right now adapt to issues emerging from the collective separating innate nature (cold beginning, banana issue, huge number of clients to run, and so on.). The arrangement introduced right now the previously mentioned issues by proposing a mixture picture recommender framework, which joins communitarian separating (social procedures) with based on the procedures. Recommender frameworks are implies for web personalization and fitting the perusing experience to the clients' particular needs. There are two classes of recommender frameworks; memory-based and model-based frameworks. Right now propose a customized recommender framework for the following page forecast that depends on a half and half model from the two classifications. The summed up designs created by a model based strategies are customized to explicit clients by incorporating client profiles produced from the conventional memory-based framework's client thing grid. The recommended framework offered a noteworthy improvement in expectation speed over conventional model-based utilization mining frameworks, while likewise offering a normal improvement in the framework exactness and framework accuracy by $0.27 \%$ and $2.35 \%$, individually.

\section{PROPOSED METHODOLOGY}

Recommender System (RS): Special kind of data sifting framework that gives an expectation that helps the client in assessing things from a huge assortment that the client is probably going to discover fascinating or valuable.

- Status update (micropost): Short message, partook in an online social stage, communicating a movement, perspective or conclusion.

- Folksonomy: Whole arrangement of labels that establishes an unstructured cooperative information order plot in a social labeling framework.

- This step includes recognizing and removing those particular item includes and the suppositions on them.

- The point of the venture is to mingle the closeout with the goal that individuals from far and wide and even over the landmass can take an interest in it.

\section{IV SYSTEM ARCHITECTURE}

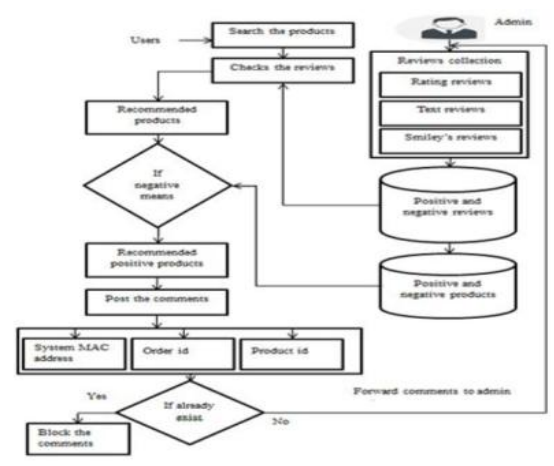

Fig 1: System Architecture

\section{MODULES}

- Collection of User's Reviews

- $\quad$ Pre-Processing

- Feature Selection

- Sentiment Word Identification

- Sentiment Polarity Identification

- Sentiment Classification

- Analysis of Reviews

\section{WORKING MODEL}

Modules Description

Assortment of User's Reviews

Surveys are vital for doing the Sentiment Analysis Task. For the Collection of audits there are various strategies which are utilized right now. The surveys can be an organized, semi-organized and unstructured sort. Opinion Analysis inquire about, there are open source system where specialist can get their information for the examination reason.By introducing required bundles and verification procedure of social site, to slither the audits from that site is simple undertaking. When we have our content information with us then we can utilize that information for Pre-handling reason.

\section{Pre-Processing}

In pre-handling following are a few assignments:

- Removing URLs, Special characters, Numbers, Punctuations and so on.

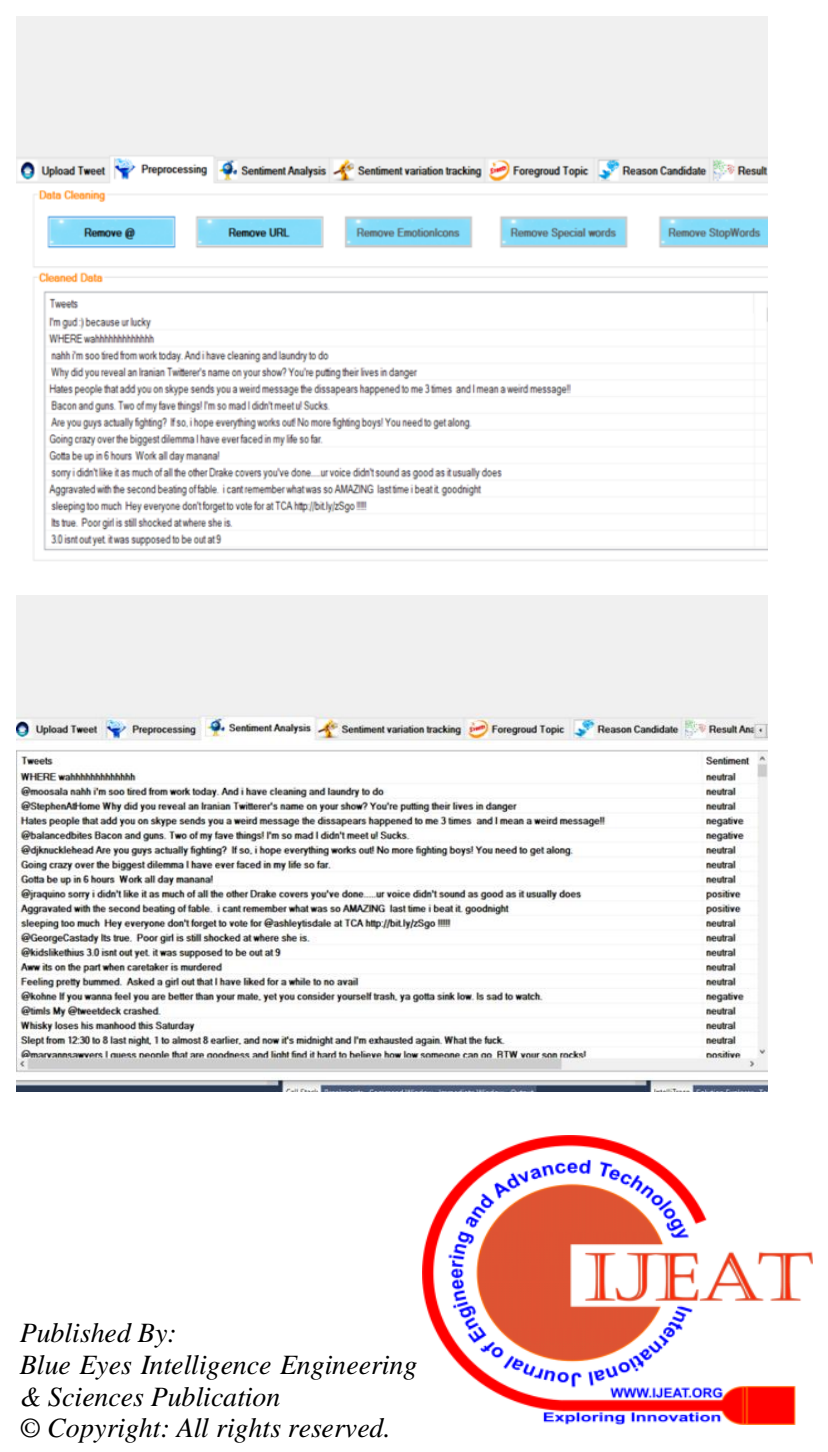




\section{The Sentimental Analysis for E-Commerce Application}

- Removing Stop words

- Removal of Retweets (in the event of twitter dataset)

- Stemming

- Tokenization

\section{Highlight Selection}

Highlight choice from pre-handled content is the troublesome errand in estimation investigation. The fundamental objective of the component choice is to diminish the dimensionality of the element space and in this way computational expense. Highlight choice will decrease the over fitting of the learning plan to the preparation information. In various AI calculations were broke down on a news audit dataset with various element choice procedures highlights are generally unigrams, bigrams and grams. POS labeling is utilized in include choice procedures.

Supposition Word Identification

Supposition word recognizable proof is an essential work in various uses of conclusion investigation and feeling mining, for example, survey mining, assessment holder finding, and audit grouping. Assumption words can be ordered into positive, negative and unbiased words.

\section{Supposition Polarity Identification}

The fundamental undertaking in SA is characterizing the extremity of a given book at the report, sentence, or highlight. The extremity is in three classification for example Positive, Negative and Neutral. Extremity distinguishing proof is finished by utilizing various vocabularies which help to ascertain conclusion score, slant quality and so forth.

\section{Opinion Classification}

Opinion arrangement of news audit dataset and item survey dataset is finished utilizing managed AI approaches like credulous Bayes, SVM, Maximum Entropy and so on. Precision is relies upon which dataset is utilized for which order techniques. On account of Supervised AI approaches Training dataset is utilized to prepare the characterization model which at that point help to order the test information. Investigation of Reviews

At long last Analysis of result is critical to settle on choice to individual and industry. If there should be an occurrence of news surveys on the off chance that more outcome is certain, at that point client can choose to go that news occasion. Investigation is utilized in business knowledge.

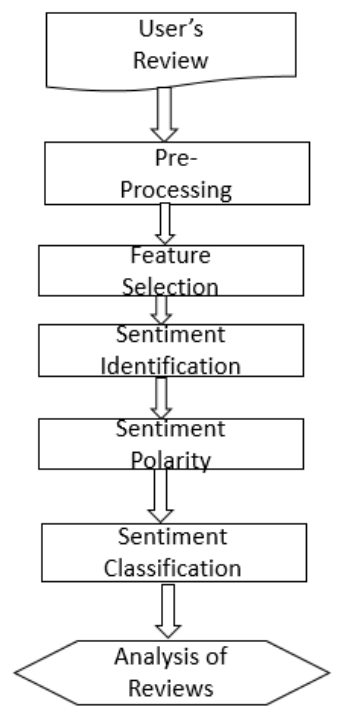

Fig 2. data flow diagram

\section{ER DIAGRAMS}

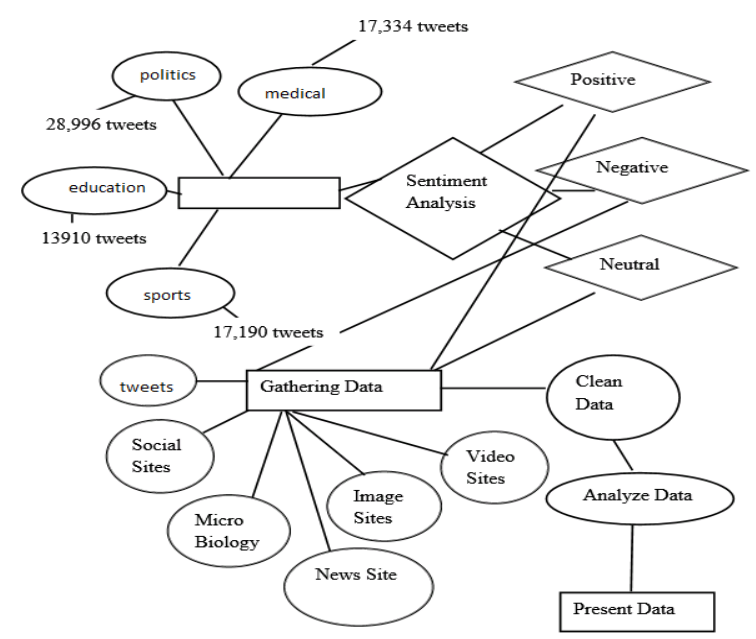

Fig 3:ER Diagram for Review analysis

\section{UML DIAGRAM}

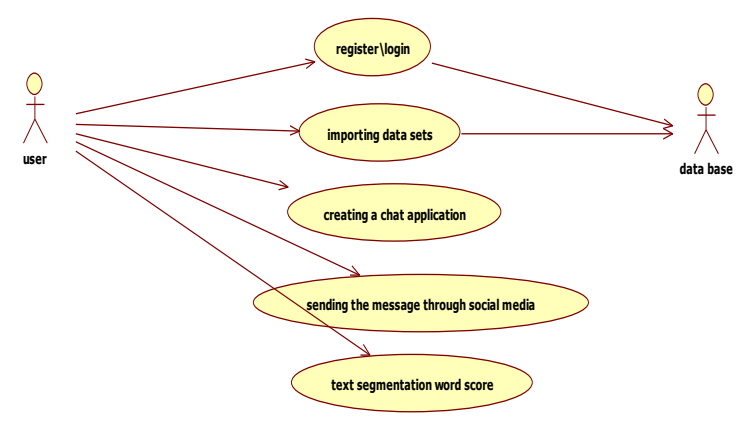

Fig 4: USE CASE DIAGRAM 


\section{CONCLUSION}

Right now, we utilized Twitter API utilizing R device which is open source. Tweets from twitter has been gathered and provides for pre-handling task in that instrument. $\mathrm{R}$ open source device is utilized in content mining and furthermore to slither gushing information from web based life like twitter and Facebook and so forth. Film surveys information additionally pre-handled in $\mathrm{R}$ instrument for feeling examination and conclusion mining. There are distinctive regulated and solo methodologies and various vocabularies, word references and corpus based techniques which are exceptionally useful in Sentiment Analysis. Distinctive dataset are accessible for film audit, item survey, dataset and so forth. Right now score has been determined and checked number of positive, negative and nonpartisan tweets for given Hashtag and can foresee the popular assessment of specific occasion. According to above investigation of various \#Hashtags tweets for estimation examination, individual and industry can locate the popular supposition behind that occasion.

\section{REFERENCES:}

1. A. S. E, "Studies of independence and conformity: I. a minority of one against a unanimous majority," Psychological monographs: General and applied, vol. 70(9), p. 1, 1956.

2. M. L. S. D. X. W. L. S. Mingliang Chen, Qingguo Ma, "The neural and psychological basis of herding in purchasing books online: an event-related potential study," Cyberpsychology, Behavior, and Social Networking, vol. 13(3), pp. 321-328, 2010.

3. N. V. Nielsen, "E-commerce: Evolution or revolution in the fastmoving consumer goods world," nngroup. com, 2014.

4. [4] B. W. O, "Reference group influence on product and brand purchase decisions," Journal of Consumer Research, vol. 9, pp. 183194, 1982.

5. A. S. E, "Studies of independence and conformity: I. a minority of one against a unanimous majority," Psychological monographs: General and applied, vol. 70(9), p. 1, 1956.

6. R. Peres, E. Muller, and V. Mahajan, "Innovation diffusion and new product growth models: A critical review and research directions,' International Journal of Research in Marketing, vol. 27, no. 2, pp. 91 $-106,2010$.

7. L. A. Fourt and J. W. Woodlock, "Early prediction of market success for new grocery products." Journal of Marketing, vol. 25, no. 2, pp. $31-38,1960$.

8. R.D.Luce, "Individual choice behavior a theoretical analysis," in john Wiley and Sons, 1959.

9. J. J. McAuley, C. Targett, Q. Shi, and A. van den Hengel, "Imagebased recommendations on styles and substitutes," in SIGIR, 2015, pp. 43-52.

10. Y. B. Xavier Glorot, "Understanding the difficulty of training deep feedforward neural networks," in AISTATS, 2010, pp. 249-256.

11. J. McAuley and A. Yang, "Addressing complex and subjective product-related queries with customer reviews," in WWW, 2016, pp. 625-635.

12. Dr.C K Gomathy, Article: A Semantic Quality of Web Service Information Retrieval Techniques Using Bin Rank A Cloud Monitoring Framework Perform in Web Services, International Journal of Scientific Research in Computer Science Engineering and Information Technology IJSRCSEIT | Volume 3 | Issue 5 | ISSN : 2456-3307,May-2018

13. Dr.C K Gomathy, Article: Supply chain-Impact of importance and Technology in Software Release Management, International Journal of Scientific Research in Computer Science Engineering and Information Technology ( IJSRCSEIT ) Volume 3 | Issue 6 | ISSN : 2456-3307, P.No:1-4, July-2018

14. Dr.C K Gomathy, Article: A Semantic Quality of Web Service Information Retrieval Techniques Using Bin Rank A Cloud Monitoring Framework Perform in Web Services, International Journal of Scientific Research in Computer Science Engineering and Information Technology IJSRCSEIT | Volume 3 | Issue 5 | ISSN : 2456-3307,May-2018
15. Dr.C K Gomathy, Article: A Web Based Platform Comparison by an Exploratory Experiment Searching For Emergent Platform Properties, IAETSD Journal For Advanced Research In Applied Sciences, Volume 5, Issue 3, P.No-213-220, ISSN NO: 2394-8442,Mar/2018

16. C K Gomathy and V Geetha. Article: Evaluation on Ethernet based Passive Optical Network Service Enhancement through Splitting of Architecture. International Journal of Computer Applications 138(2):14-17, March 2016. Published by Foundation of Computer Science (FCS), NY, USA, ISSN No: 0975-8887

17. C K Gomathy and V Geetha. Article: A Real Time Analysis of Service based using Mobile Phone Controlled Vehicle using DTMF for Accident Prevention. International Journal of Computer Applications 138(2):11-13, March 2016. Published by Foundation of Computer Science (FCS), NY, USA,ISSN No: 0975-8887

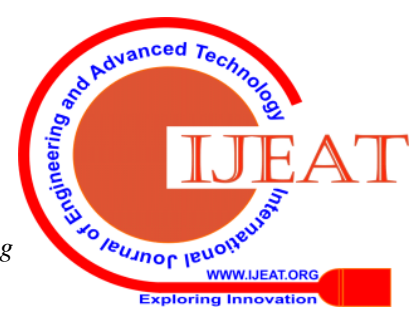

